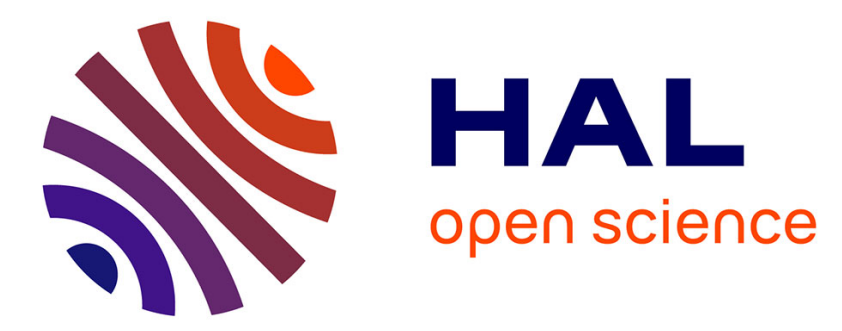

\title{
Chipless RFID Tag Discrimination and the Performance of Resemblance Metrics to be used for it
}

Zeshan Ali, Nicolas Barbot, Romain Siragusa, David Hely, Maxime Bernier, Frédéric Garet, Etienne Perret

\section{- To cite this version:}

Zeshan Ali, Nicolas Barbot, Romain Siragusa, David Hely, Maxime Bernier, et al.. Chipless RFID Tag Discrimination and the Performance of Resemblance Metrics to be used for it. IEEE/MTT-S International Microwave Symposium - IMS 2018, Jun 2018, Philadelphia, United States. 10.1109/MWSYM.2018.8439855 . hal-01888533

\section{HAL Id: hal-01888533 https://hal.science/hal-01888533}

Submitted on 13 Jan 2020

HAL is a multi-disciplinary open access archive for the deposit and dissemination of scientific research documents, whether they are published or not. The documents may come from teaching and research institutions in France or abroad, or from public or private research centers.
L'archive ouverte pluridisciplinaire HAL, est destinée au dépôt et à la diffusion de documents scientifiques de niveau recherche, publiés ou non, émanant des établissements d'enseignement et de recherche français ou étrangers, des laboratoires publics ou privés. 


\title{
Chipless RFID Tag Discrimination and the Performance of Resemblance Metrics to be used for it
}

\author{
Zeshan Ali ${ }^{1}$, Nicolas Barbot ${ }^{1}$, Romain Siragusa ${ }^{1}$, David Hely ${ }^{1}$, Maxime Bernier ${ }^{2}$, Frédéric Garet $^{2}$, \\ and Etienne Perret ${ }^{1,3}$ \\ ${ }^{1}$ Univ. Grenoble Alpes, Grenoble INP, LCIS, 26000 Valence, France \\ ${ }^{2}$ Univ. Savoie Mont Blanc, CNRS, Grenoble INP, IMEP-LAHC, 73376 Le Bourget-du-Lac, France \\ ${ }^{3}$ Institut Universitaire de France, 75005 Paris, France \\ zeshan.ali@1cis.grenoble-inp.fr
}

\begin{abstract}
This paper proposes a novel radar based approach for chipless tag discrimination. The proposed technique can be considered as a first step towards chipless authentication of manufactured products. The concept of chipless radiofrequency identification (RFID) is extended to tag discrimination, where each tag produces a unique signature that is very difficult to reproduce even if a clone of the device is made. The proposed technique, using radiofrequency $(\mathrm{RF})$ electromagnetic (EM), keeps non-invasive and non-destructive. This new method to discriminate tag's RF signature is introduced based on the comparison between two resemblance metrics in the frequency domain and time domain. To calculate the resemblance between different signatures entire part of the RF signatures is exploited to utilize the maximum signals' richness. Owing to the chipless RF approach, we show that geometrical variations less than $10 \mu \mathrm{m}$ (i.e., smaller than $\lambda / \mathbf{1 0 0 0}$ ) can be detected which demonstrate the extreme sensitivity of the method.
\end{abstract}

Index Terms-Authentication technology, chipless RFID, fabrication tolerance, radar approach, randomness.

\section{INTRODUCTION}

Chipless radio frequency (RF) identification (RFID), also known as RF barcode, has already emerged as a potential tool for identification applications [1]. Recently, chipless RFID is also evolving towards authentication applications. In the same idea, an unclonable concentric ring slot resonators based chipless RFID tag has been presented recently [2]. In this method, authentication based on simulation results is discussed, where supposed randomness along numerous slot parameters (air gap, trace width, substrate thickness, and dielectric constant of substrate) is considered and the uniqueness of the tags is measured by calculating the Euclidean distance between the resonance frequencies. In [3], a RFID authentication system has been presented. Near field RF signatures from various RF certificates of authenticity (RF-CoA) are exploited and the uniqueness of the CoAs is analyzed by using standard deviation of received signal strength from the different antennas for various CoAs at the same frequency points and the antenna couplings. Otherwise, a tag-less RFID technique has been demonstrated to classify the electronic devices [4], using directly radiated RF signals by the device itself. This technique is based on the comparison of the EM radiations of the given electronic devices with others considered as reference ones, using cosine similarity function.

This paper discusses the capability to discriminate the chipless tags based on minor dimensional variations by using chipless RFID technology. Our objective is to introduce different resemblance metrics that could be promising to differentiate RF signatures and then to conclude that the process variations $\Delta P$ inherent to the fabrication process are sufficient to produce unique RF signatures. Section II is dedicated to the design of the chipless tags and their measurement setup. Section III validates the proposed method by doing freespace based tag discrimination in both the frequency domain (FD) and time domain (TD), where the performance of two resemblance metrics is compared. Finally, Section IV presents the conclusions.

\section{Chipless Tag and Variations Along Geometric} Dimensions

The C-folded dipole has been extensively used in the design and development of chipless RFID tags [1]. The response of such a tag is mainly driven by two key dimension parameters [see Fig. 1(a)]: the spacing between the two arms $g$ and the length $L^{\prime}$. The frequency of resonance $f_{r}$ can be then the written:

$$
f_{r}=\frac{c}{4 L^{\prime} \sqrt{\varepsilon_{e f f}}}
$$

where, $L^{\prime}$ is the total length which is the sum of $L$ (physical length of each arm) and $\Delta L$ (complementary length added due to the fringing fields), and $\varepsilon_{e f f}$ is the effective permittivity of the substrate for a coplanar strip line [5]. Each fabricated circuit being altered by $\Delta P$ exhibits possible distinct signature. To evaluate the uniqueness of a tag at least two measurements are needed: one for the database and the other for the examination. The $\Delta P$ is an unknown random variable that critically depends on the technology chosen for circuit realization. For conventional chemical etching process, this unknown $\Delta P$ is of the order of the metal thickness $t$. In this paper, Rogers RO3003 substrate is used having $\varepsilon_{r}=3, h=0.75 \mathrm{~mm}$, and $t=35 \mu \mathrm{m}$. For a $\mathrm{C}$-folded scatterer exhibiting dimension parameters: $w=2 \mathrm{~mm}, g=0.8 \mathrm{~mm}$, and $L^{\prime}=13.2 \mathrm{~mm}$, it is calculated that $\varepsilon_{\text {eff }}=1.2795$ and $f_{r}=5.023 \mathrm{GHz}$. As $t=35 \mu \mathrm{m}$, it is assumed that the maximum probable variation along the geometric dimensions is $35 \mu \mathrm{m}$. If we impose an assumed maximum variation of $35 \mu \mathrm{m}$ merely along the length, a significant shift of $13.4 \mathrm{MHz}$ in the frequency of resonance $f_{r}$ can be calculated using (1). It is important to note that the frequency of resonance $f_{r}$ of the scatterer is not directly 

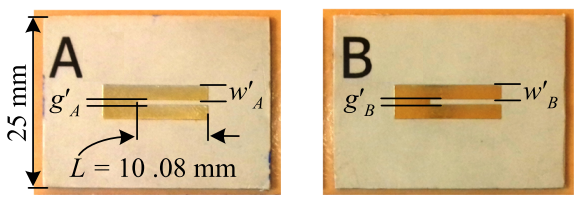

(a)

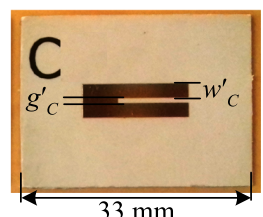

$33 \mathrm{~mm}$

TABLE I

Dimension Parameters of the Three Fabricated Tags.

\begin{tabular}{|c|c|c|}
\hline \multirow{2}{*}{ Tags } & \multicolumn{2}{|c|}{ Dimension parameters (mm) } \\
\cline { 2 - 3 } & $w^{\prime}$ & $g^{\prime}$ \\
\hline $\mathrm{A}$ & $w+\Delta P$ & $g+\Delta P$ \\
$\mathrm{~B}$ & $w+0.0175+\Delta P$ & $g-0.0175+\Delta P$ \\
$\mathrm{C}$ & $w+0.035+\Delta P$ & $g-0.035+\Delta P$ \\
\hline
\end{tabular}

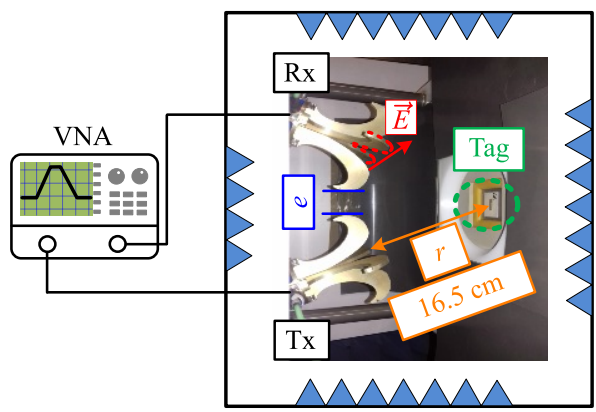

(b)

Fig. 1. (a) Top view of fabricated C-folded tags. (b) Measurement setup in anechoic environment.

linked to the $g$ and $w$. The focus of this study is to examine the variations along these latter parameters (except $L^{\prime}$ ), which have less effect on the frequency of resonance $f_{r}$. In this way, if we impose an assumed maximum variation on these two dimensions $w=2+0.035 \mathrm{~mm}, g=0.8-0.035 \mathrm{~mm}$ and keeping the $L^{\prime}$ fixed, we deduce that $\varepsilon_{\text {eff }}=1.2821$ and $f_{r}=5.018 \mathrm{GHz}$. This operation leads to a slight shift in the frequency of resonance $f_{r}$ around an initial value of $5 \mathrm{MHz}$. Note that these variations would not merely affect the $f_{r}$, but more generally all the data points of the RF signature.

To experimentally validate the method, three tags have been realized [see Fig. 1(a)]. Tag A exhibits the nominal theoretical geometric dimensions, for example, $L=10.08 \mathrm{~mm}, w=2 \mathrm{~mm}$, and $g=0.8 \mathrm{~mm}$, designed for a resonance frequency of $5 \mathrm{GHz}$. In tag $\mathrm{B}$, the spacing between the two arms $g$ is decreased and the width of the trace $w$ is increased by a purposely applied theoretical variation of $17.5 \mu \mathrm{m}$, whereas these theoretical variations are double for the tag $\mathrm{C}$. This work is based on the purposely applied variations, so the unknown $\Delta P$ happening during the fabrication process are considered as a part of the applied variations. The purpose behind such applied variations is to prove the concept by introducing proper analytical treatment that can quantify these very slight variations without ambiguity. Table I shows the dimension parameters for all three fabricated tags, where primed parameters $\left(w^{\prime}\right.$ and $g^{\prime}$ ) are the dimension parameters including the effect of the unknown $\Delta P$. It is important to mention that each entry of $\Delta P$ in Table I shows a distinct and unknown process variation. Subsequently, we carefully measured the scatterers' dimensions by optical microscope to consider the unknown $\Delta P$. These dimensions are measured at the two ends of each arm of the scatterer. The average values of the measured dimensional variations among three tags in comparison to the theoretical applied variations are outlined in Table II, where the contribution of $\Delta P$ can be observed. It can be observed that

for both $w^{\prime}$ and $g^{\prime}$ parameters among three tags, the minimum measured average dimensional changes are $\Delta w_{B C}^{\prime}=7.89 \mu \mathrm{m}$ and $\Delta g_{A B}^{\prime}=4.6 \mu \mathrm{m}$. All the other geometric dimensions (e.g., $L$ ) of the C-folded dipole are the same for all the patterns, as well as the overall size of the tags: $33 \times 25 \mathrm{~mm}^{2}$.

Fig. 1(b) shows measurement setup in an anechoic environment with two antennas, keeping a spacing of $e=2.7 \mathrm{~cm}$ while the tag is placed at a distance of $r=16.5 \mathrm{~cm}$ from both antennas. The experimental results are measured using a vector network analyzer (VNA; Agilent 5222A) with a source power of $-5 \mathrm{dBm}$. All three tags are successively measured fifteen times, where each measurement is done by removing and repositioning the tag at its position. Although, measurements are carried out in a bistatic co-polarization configuration, we present only the results of reflection coefficient $S_{11}$ which seem to be more favorable for practical case due to the requirement of fewer resources (one antenna). A background normalization of $S$-parameters is carried out such that $S^{\text {tag }}=S^{r a w}-S^{b a c k}$, where $S^{r a w}$ and $S^{\text {back }}$ are the measurements in the presence and the absence of the tag, respectively. The FD and TD backscattered $S_{11}$ of tag A are shown in Fig. 2. Middle part of the TD signal is extracted by applying time windowing of a time duration of $40 \mathrm{~ns}$ [Fig. 2(b)]. This time windowing is carried by calculating IFFT of measured FD signal (nonwindowed signals, solid red line), applying truncation by zero padding in TD, and calculating FFT of truncated TD signal (windowed signals, dashed blue line). The elimination of $15 \mathrm{~ns}$ of early part of the TD signal is carried out to discard the direct reflections from the tag and its holder (the structural mode). These high reflections from the tag's holder are due to its solid plastic material. This solid holder is used to ensure the exact position in each measurement trial. For the rest of this paper, we take into account the windowed signals for the similarity analysis, where both the FD and the TD windowed signals exhibit 8001 data points. 


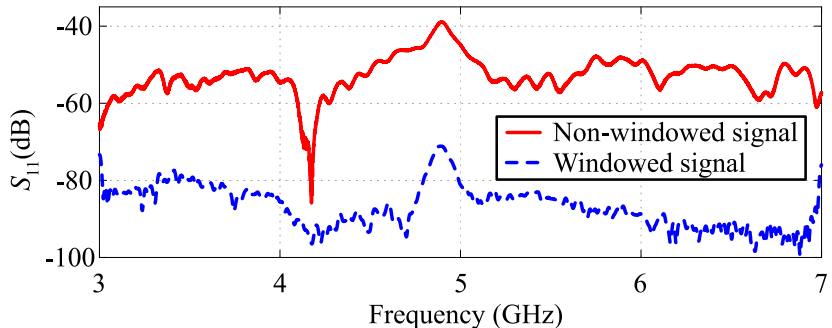

(a)

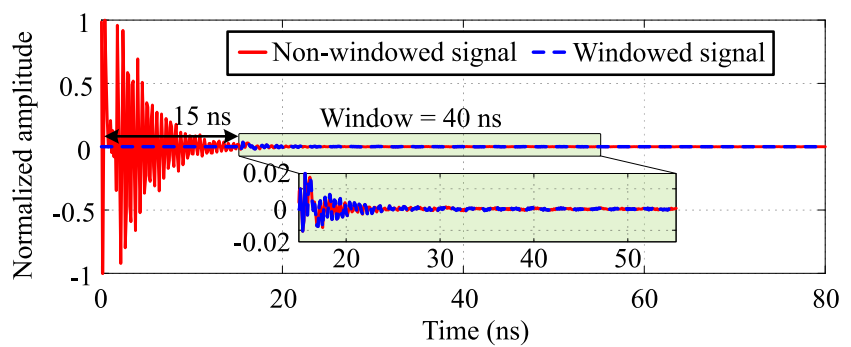

(b)

Fig. 2. Reflection coefficient $S_{11}$ of the tag A. (a) Frequency domain responses. (b) Time domain responses.

\section{Tag Discrimination Results and Performance of the Resemblance Metrics}

As for one tag, the total number of repetitive measurements is 15 , the possible number of inter-tag combinations between two different tags is $15 \times 15=225$ and the possible number of intra-tag combinations to compare all repetitive measurements for each tag is $C_{2}^{15}=105$. The level of similarity for all the combinations is calculated in two types of domains: FD and TD.

In the FD, we used cosine similarity (CS) to evaluate the similarity of the different data sets. If we consider two EM signals, having complex and discrete spectrums $\mathrm{u}=\left[u_{1}, u_{2}, \ldots, u_{n}\right]$ and $\mathrm{v}=\left[v_{1}, v_{2}, \ldots, v_{n}\right]$, the mathematical expression of CS writes:

$$
\mathrm{CS}=\left|\frac{\sum\left(\mathrm{u}_{i} \cdot \mathrm{v}_{i}^{*}\right)}{\sqrt{\sum\left(\mathrm{u}_{i}\right)^{2}} \sqrt{\sum\left(\mathrm{v}_{i}\right)^{2}}}\right|
$$

where, operator $*$ denotes the conjugate of the complex entry. The intra- and inter-tag CS distributions are presented in Fig. 3(a). The margin between the minimum intra-tag CS and the maximum inter-tag $\mathrm{CS}$ is approximately equal to 0.072 . In TD analysis, maximum value of correlation coefficient $\left(\mathrm{CC}_{\max }\right)$ is used as a similarity metric. Considering two time series corresponding, for example, to backscattered EM signals obtained from discrete spectrums $\mathrm{n}=\left[n_{1}, n_{2}, \ldots, n_{n}\right]$ and $\mathrm{m}=\left[m_{1}, m_{2}, \ldots, m_{n}\right]$, the mathematical expression of $\mathrm{CC}_{\max }$ is:

$$
\mathrm{CC}_{\max }=\max \left[\frac{\sum_{i}\left(\mathrm{n}_{i}-\mu_{\mathrm{n}}\right)\left(\mathrm{m}_{i}-\mu_{\mathrm{m}}\right)}{\sqrt{\sum_{i}\left(\mathrm{n}_{i}-\mu_{\mathrm{n}}\right)^{2}} \sqrt{\sum_{i}\left(\mathrm{~m}_{i}-\mu_{\mathrm{m}}\right)^{2}}}\right]
$$

where, $\mu_{\mathrm{n}}$ and $\mu_{\mathrm{m}}$ are the arithmetic means of the two time series EM signals, respectively. Fig. 3(b) illustrates the

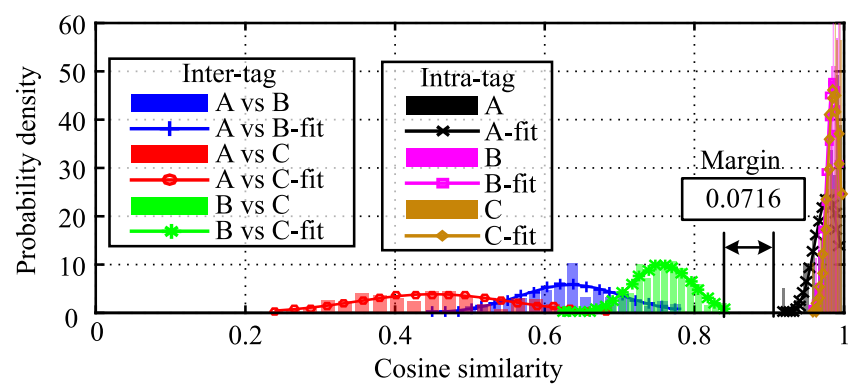

(a)

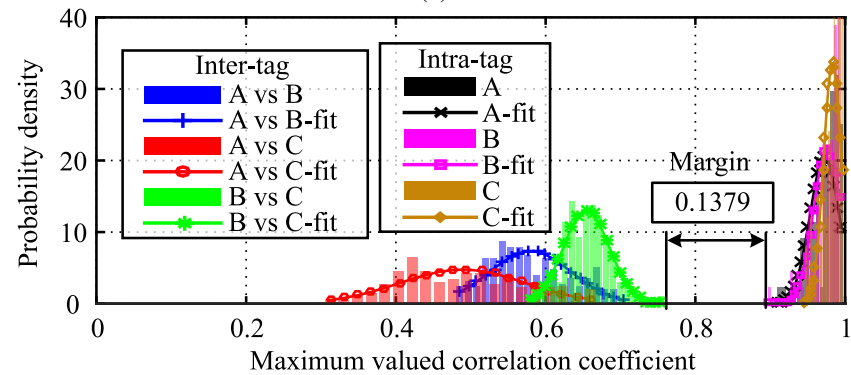

(b)

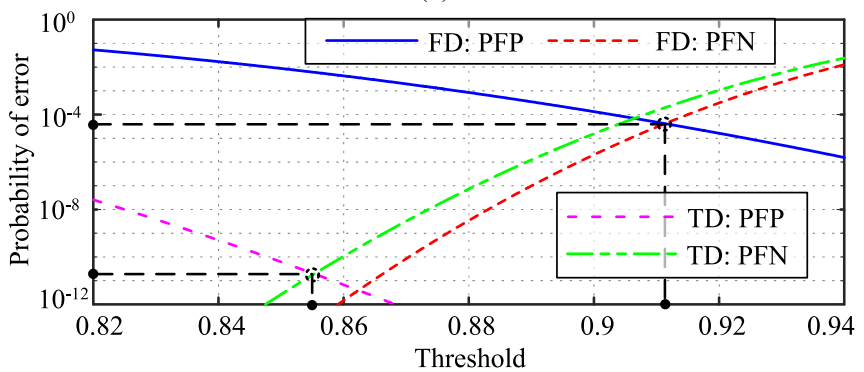

(c)

Fig. 3. (a) Intra- and inter-tag cosine similarity distributions for FD analysis (b) Intra- and inter-tag maximum valued correlation coefficient distributions for TD analysis. (c) Probabilities of false positive and false negative for both FD and TD analyses.

intra- and inter-tag $\mathrm{CC}_{\max }$ distributions calculated on all the backscattered signals of the three tags. The margin between the maximum inter-tag $\mathrm{CC}_{\max }$ and the minimum intra-tag $\mathrm{CC}_{\max }$ is of about 0.138 . The central tendency, for example, mean value of the intra-tag distributions of both FD and TD analyses is close to unity [see Fig. 3(a) and (b)], which validates the repeatability of each tag. A slight spread of the intratag distributions below the unity is due to the uncertainties (systematic error and random error) from the measurement bench. On the other hand, the arrangement of the inter-tag similarity distributions of both FD and TD analyses is according to the measured average dimensional variations (see Table II).

We calculated the probability of false negative (PFN) and probability of false positive (PFP) by using the fitted Gaussian probability density functions [see Fig. 3(a) and (b)] for both FD and TD analyses. The error rates are presented in Fig. 3(c). For the FD, we found a probability of error (PE) lower than $10^{-4}$, when the closest distributions are chosen (i.e., the worst case): inter-tag $\mathrm{B}$ vs $\mathrm{C}$ distribution and intra-tag A distribution. While, in the TD, the PE is lower than $10^{-10}$, when the closest 
distributions inter-tag $\mathrm{B}$ vs $\mathrm{C}$ distribution and intra-tag $\mathrm{B}$ distribution are chosen (i.e., also a worst case).

Concerning the performance of similarity measures, the $\mathrm{CC}_{\max }$ outperforms the $\mathrm{CS}$ as the margin is almost two-fold in TD analysis as compared to FD analysis (see Fig. 3). In general, both similarity measures $\left(\mathrm{CS}\right.$ and $\mathrm{CC}_{\max }$ ) are robust. However, from a practical view point where data acquisition is done in FD using a VNA, CS seems promising. Furthermore, as $\mathrm{CS}$ is an absolute valued normalized inner product between two complex vectors, it is less time consuming as compared to $\mathrm{CC}_{\max }$, where the correlation coefficient is calculated at each point by passing two real valued vectors from each other and maximum value is taken. On the other hand, $\mathrm{CC}_{\max }$ being insensitive to the time delay in TD is also an efficient and robust alternative.

In [2], a complex multi-resonant structure is used and the sum of the corresponding Euclidean distances among the multiple frequencies of resonance is used to distinguish the tags. While, in this work, a simple single-resonant structure is used and the all the data points of the spectrum in both $\mathrm{CS}$ and $\mathrm{CC}_{\max }$ are utilized to discriminate the tags. Hence, the performance of the both $\mathrm{CS}$ and $\mathrm{CC}_{\max }$ is good even if the contribution of the measurement bench is included (i.e., all the data points of the spectrum).

The concept discussed in this work can be applied on a larger number of tags having multi-scatterers realized with natural process variations for chipless RF authentication.

\section{Conclusion}

In this study, the capabilities of chipless technology to be used for tag discrimination were demonstrated. To validate the proposal, three chipless RFID tags were realized. Consecutively from one tag to another, a variation (in the order of fabrication tolerance) was applied purposely to the geometric dimensions exhibiting the lowest impact on the signal. Chipless tag discrimination based on the level of similarity was presented in both the frequency and time domains. These analyses show quite smaller values of probability of error even if the worst cases of intra- and inter-tag distributions were chosen. It is found that chipless RFID is capable of discriminating practically two different tags affected by the similar geometrical variations that are inherent to the fabrication process and in future it might be extended to authentication applications.

\section{ACKNOWLEDGMENT}

The authors would like to acknowledge the University of Grenoble Alpes for financially supporting this project via the AGIR program. The authors are also grateful to the Région Auvergne-Rhône-Alpes for supporting this project via the ARC6 program. The authors would also like to extend their deepest gratitude to the Institut Universitaire de France for supporting this project.

\section{REFERENCES}

[1] E. Perret, Radio Frequency Identification and Sensors: From RFID to Chipless RFID. John Wiley \& Sons, 2014.

[2] K. Yang, D. Forte, and M. M. Tehranipoor, "UCR: An unclonable chipless RFID tag," in Proc. IEEE Int. Symp. Hardw. Oriented Secur. Trust (HOST), Washington, DC, USA, 2016, pp. 7-12.

[3] V. Lakafosis et al., "RF fingerprinting physical objects for anticounterfeiting applications," IEEE Trans. Microw. Theory Tech., vol. 59, no. 2, pp. 504-514, 2011

[4] C. Yang and A. P. Sample, "EM-ID: Tag-less identification of electrical devices via electromagnetic emissions," in IEEE Int. Conf. RFID, Florida, MA, USA, 2016, pp. 1-8.

[5] R. Garg, I. Bahl, and M. Bozzi, Microstrip Lines and Slotlines, Third Edition. Artech House, 2013. 